\title{
Interactive granular computing
}

\author{
Andrzej Skowron ${ }^{1,2} \cdot$ Andrzej Jankowski ${ }^{3}$ Soma Dutta ${ }^{1,4}$
}

Received: 23 July 2015/ Accepted: 16 November 2015/Published online: 5 January 2016

(c) The Author(s) 2015. This article is published with open access at Springerlink.com

\begin{abstract}
Decision support in solving problems related to complex systems requires relevant computation models for the agents as well as methods for reasoning on properties of computations performed by agents. Agents are performing computations on complex objects [e.g., (behavioral) patterns, classifiers, clusters, structural objects, sets of rules, aggregation operations, (approximate) reasoning schemes]. In Granular Computing ( $\mathrm{GrC}$ ), all such constructed and/or induced objects are called granules. To model interactive computations performed by agents, crucial for the complex systems, we extend the existing $\mathrm{GrC}$ approach to Interactive Granular Computing (IGrC) approach by introducing complex granules (c-granules or granules, for short). Many advanced tasks, concerning complex systems, may be classified as control tasks performed by agents aiming at achieving the high-quality computational trajectories relative to the considered quality measures defined over the trajectories. Here, new challenges are to develop strategies to control, predict, and bound the behavior of the system. We propose to investigate these challenges using the IGrC
\end{abstract}

\footnotetext{
Andrzej Skowron

skowron@mimuw.edu.pl

Andrzej Jankowski

andrzej.adgam@gmail.com

Soma Dutta

somadutta9@gmail.com

1 Institute of Mathematics, Warsaw University, Banacha 2, 02-097 Warsaw, Poland

2 Systems Research Institute, Polish Academy of Sciences, Newelska 6, 01-447 Warsaw, Poland

3 The Dziubanski Foundation of Knowledge Technology, Nowogrodzka 31, 00-511 Warsaw, Poland

4 Vistula University, Stoklosy 3, 02-787 Warsaw, Poland
}

framework. The reasoning, which aims at controlling of computations, to achieve the required targets, is called an adaptive judgement. This reasoning deals with granules and computations over them. Adaptive judgement is more than a mixture of reasoning based on deduction, induction and abduction. Due to the uncertainty the agents generally cannot predict exactly the results of actions (or plans). Moreover, the approximations of the complex vague concepts initiating actions (or plans) are drifting with time. Hence, adaptive strategies for evolving approximations of concepts are needed. In particular, the adaptive judgement is very much needed in the efficiency management of granular computations, carried out by agents, for risk assessment, risk treatment, and cost/benefit analysis. In the paper, we emphasize the role of the rough set-based methods in IGrC. The discussed approach is a step towards realization of the Wisdom Technology (WisTech) program, and is developed over years, based on the work experience on different real-life projects.

Keywords Rough set - (Interactive) granular computing · Interactive computation - Adaptive judgement - Efficiency management · Risk management - Cost/benefit analysis . Big data technology - Cyber-physical system · Wisdom web of things · Ultra-large system

\section{Introduction}

GrC has emerged from many different disciplines and fields, including General Systems Theory, Hierarchy Theory, Social Networks, Artificial Intelligence (AI), Human Problem Solving, Learning, Programming, Theory of Computation, and Information Processing (Yao 2008). In recent years, one can observe a growing interest in the area 
of $\mathrm{GrC}$ as a methodology for modeling and conducting complex computations, in various domains of $\mathrm{AI}$ and Information Technology (IT). In particular, GrC brings a very natural methodology for problem solving in AI. Complex systems are becoming more and more important for applications in IT. Ultra-Large-Scale (ULS) systems (Cyber-physical and ultra-large-scale systems 2013) are some among them. ULS systems are interdependent webs consisting of software-intensive systems, people, policies, cultures, and economies. ULS are characterized to have properties such as: (i) decentralization, (ii) inherently conflicting, unpredictable, and diverse requirements, (iii) continuous evolution and deployment, (iv) heterogeneous, inconsistent, and changing elements, (v) erosion of the people/system boundary, and (vi) routine failures (Cyberphysical and ultra-large-scale systems 2013). Cyber-Physical Systems (CPSs) (Lamnabhi-Lagarrigue et al. 2014) and/or systems based on Wisdom Web of Things (W2T) (Zhong et al. 2013) can be treated as special cases of ULS. It is predicted that applications based on the above-mentioned systems will have enormous societal impact and economic benefit. However, there are many challenges related to such systems. In this article, we claim that further development of such systems should be based on the relevant computation models.

There are several important issues which should be taken into account in developing such computation models. Among them some are as follows. (i) Computations are performed on complex objects with very different structures, where the structures themselves are constructed and/or induced from data and domain knowledge. (ii) Computations are performed in an open world and they depend on the interactions of physical objects. (iii) Due to uncertainty, the properties and results of interactions can be perceived by agents only partially. (iv) Computations are realized in the societies of interacting agents including humans. (v) Agents are aiming at achieving their tasks by controlling computations. (vi) Agents can control computations using adaptive judgement, in which all of deduction, induction and abduction are used.

We propose to base our approach on the relevant computation model of IGrC framework, proposed recently as an extension of the GrC. The label Granular Computing was suggested by T. Y. Lin in late 1990s.

Granulation of information is inherent in human thinking and reasoning processes. It is often realized that precision is sometimes expensive and not very meaningful in modeling and controlling complex systems. When a problem involves incomplete, uncertain, and vague information, it may be difficult to discern distinct objects, and one may find it convenient to consider granules for tackling the problem of concern. Granules are composed of objects that are drawn together by indiscernibility, similarity, and/ or functionality among the objects (Zadeh 1997). Each of the granules according to its structure and size, with a certain level of granularity, may reflect a specific aspect of the problem, or form a portion of the system's domain. $\mathrm{GrC}$ is considered to be an effective framework in the design and implementation of intelligent systems for various reallife applications. The systems based on $\mathrm{GrC}$, e.g., for pattern recognition, exploit the tolerance for imprecision, uncertainty, approximate reasoning as well as partial truth of soft computing framework, and are capable of achieving tractability, robustness, and close resemblance with human-like (natural) decision-making (Bargiela and Pedrycz 2003; Pedrycz 2013; Pedrycz et al. 2008; Skowron et al. 2011).

In $\mathrm{GrC}$, computations are performed on granules of different structures, where granularity of information plays an important role. Information granules (infogranules, for short) in $\mathrm{GrC}$ are widely discussed in the literature (Pedrycz et al. 2008). In particular, let us mention here the rough granular computing approach based on the rough set approach, and its combination with other approaches to soft computing, such as fuzzy sets. However, the issues related to the interactions of infogranules with the physical world, and perception of interactions in the physical world by means of infogranules are not well elaborated yet. Understanding interactions is one of the critical issues of complex systems (Goldin et al. 2006). For example, the ULS are autonomous or semiautonomous systems, and cannot be designed as closed systems that can operate in isolation; rather, the interaction and potential interference among smart components, among CPSs, and among CPSs and humans, are required to be modeled by coordinated, controlled, and cooperative behavior of agents representing components of the system (Cyber-physical and ultra-largescale systems 2013).

We extend the existing GrC approach to IGrC by introducing complex granules (c-granules, for short) (Skowron et al. 2012; Jankowski et al. 2014) making it possible to model interactive computations carried out by agents and their teams in complex systems working in an open-world environment.

Any agent operates on a local world of c-granules. The agent aims at controlling computations performed on c-granules from this local world for achieving the target goals. In our approach, computations in systems based on IGrC proceed through complex interactions among physical objects. Some results of such interactions are perceived by agents with the help of c-granules.

The discussed approach is a step towards one way of realization of the WisTech program (Jankowski and Skowron 2007). The approach was developed over years of work on different real-life projects. 
This article is organized as follows. In Sect. 2, an introduction to IGrC is presented. In particular, we present intuitions concerning the definition of c-granules. Interactive computations on c-granules realized by agents are discussed in Sect. 3. Issues related to agent's control based on IGrC are outlined in Sect. 4. Section 5 is devoted to the issues related to decision support of users in solving problems using IGrC. This section includes comments on problem specification in IGrC (Sect. 5.1). Next, some strategies for construction and discovery of new relevant granules are presented (Sect. 5.2). In particular, such strategies can be based on (i) operations of aggregation of information systems (called as operations of joining with constraints), (ii) inducing a hierarchy of satisfiability relations, (iii) selforganization of agents, or (iv) communications and dialogue among agents. The approach to efficiency analysis, in particular to risk management, in controlling computations over granules in Big Data Technology (BDT), is outlined in Sect. 5.6.

The role of reasoning based on adaptive judgement is discussed in Sect. 6. Section 7 concludes the paper.

The paper summarizes as well as extends the work developed by Jankowski et al. (2014), Skowron and Jankowski (2015) and Skowron et al. (2012, 2015).

\section{Complex granules}

Infogranules are widely discussed in the literature (see, e.g., Skowron and Stepaniuk 2004). They can be treated as specifications of compound objects, which are defined in a hierarchical manner, together with descriptions regarding their implementations. Such granules are obtained as the result of information granulation (Zadeh 2001):

Information granulation can be viewed as a human way of achieving data compression and it plays a key role in implementation of the strategy of divide-andconquer in human problem solving.

Infogranules belong to those concepts which play the main role in developing foundations of AI, data mining, and text mining (Pedrycz et al. 2008). They grew up as some generalizations from fuzzy set theory, rough set theory, and interval analysis (Pedrycz et al. 2008). In GrC, to deal with vague concepts, rough sets, fuzzy sets, and interval analysis are used. However, the issues related to the interactions of infogranules with the physical world, and their relationship for perceiving interactions in the physical world are not well elaborated yet (Goldin et al. 2006). On the other hand, in Goldin et al. (2006), it is mentioned that:
[...] interaction is a critical issue in the understanding of complex systems of any sorts, [...] it has emerged in several well-established scientific areas other than computer science, like biology, physics, social and organizational sciences.

Interactive computations of an agent in IGrC (Jankowski and Skowron 2007; Jankowski et al. 2014; Skowron and Wasilewski 2011; Skowron et al. 2012) are realized on configurations of c-granules generated by the agent's control. Roughly speaking, the aim of any c-granule is to link relevant infogranule(s) (Pedrycz et al. 2008) with a collection (or a structure) of spatiotemporal physical objects from the agent's environment perceived through the "windows" of the c-granule. Collections (or structures) of spatiotemporal physical objects perceived by the agent through such windows are called hunks (Heller 1990; Jankowski et al. 2014; Skowron et al. 2012). The infogranules linked by the c-granule with a particular collection (or structure) of spatiotemporal physical objects represent properties of interactions of objects from this collection (or structure).

Any c-granule is synthesized with three physical components, namely soft_suit, link_suit and hard_suit.

The soft_suit component of a given c-granule consists of configurations of the hunks representing properties of the activity environment of ag; e.g., present, past, and expected results of activities of ag, as well as expected results of some potential interactions, activated by the c-granule, are parts of the soft_suit. It is used to record the properties of hunks and their interactions perceived by the c-granule.

The link_suit can be treated as a communication channel (composed out of links) transmitting results of interactions among accessible fragments of the activity environment of $\mathrm{ag}$, including also results of interactions among hunks in the soft_suite. Note that some weights may be assigned to the links reflecting the results of judgement by ag based on the priorities relative to the current hierarchy of needs of ag.

The hard_suit is composed out of configurations of hunks accessible by links from the link_suit.

The soft_suit encodes procedures for: (i) recording some properties of interactions among hunks in the hard_suit, which are transmitted to soft_suit using link_suit, and (ii) initiating relevant interactions in the hard_suit. We assume that the relevant pointers to the link_suit, hard_suit, and/or soft_suit are represented in the soft_suit (or agent's control), and make it possible to identify these components, if necessary. We also assume that the soft_suit may represent the information about the expected results of the perceived interactions, which take place in the hard_suit.

In Fig. 1, we present an illustrative c-granule at a moment $t$ of the agent's time. The results of perception are 
stored in the soft_suit, and they include information about parts of the hunk perceived at time $t$ (e.g., a pair of penguins, a piece of ice land, and a camera). The soft_suit is linked using link_suit, which plays a role of transmitting channel for interactions among the perceived hunks in the hard_suit. Figure 2 illustrates changes of 'windows' for tracking of a particular pair of penguins over time.

Interactions of the agent with the environment are realized using configurations of c-granules. Due to the interactions of c-granules with the environment (including the agent's control) the actual configuration of c-granules of an agent is evolving with local time of the agent. This leads to the changes of the existing configuration of c-granules by

- extending it to new c-granules selected by the agent's control for perceiving new interactions (also stimulated by c-granules),

- extending it to new c-granules for encoding the results of the perceived interactions,

- deleting some c-granules from the current configuration, and

- introducing other kinds of modifications of some parts of the configuration.

In general, c-granules of ag support activities such as

- improvement of techniques of representation according to her/his hierarchy of needs and perception of needs, as well as relations between them;

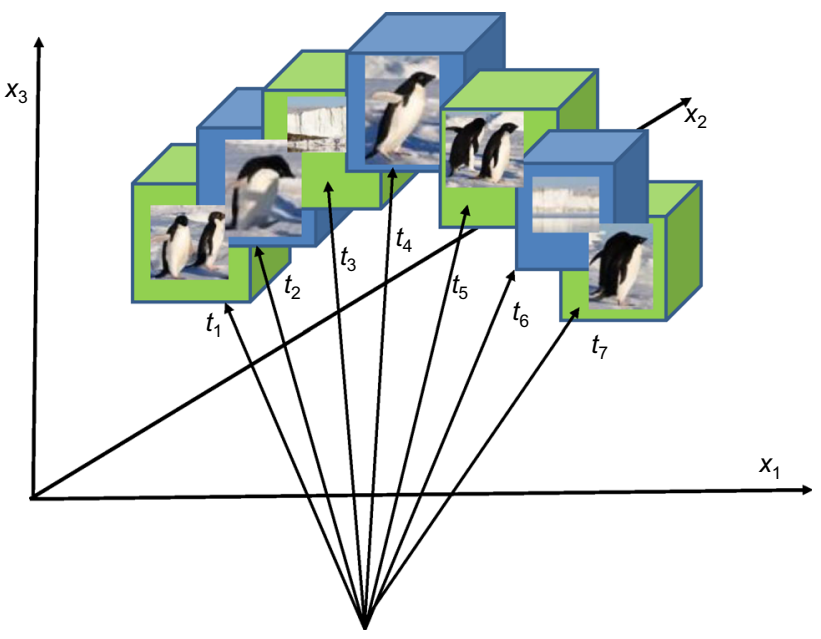

Fig. 2 Links to a (spatiotemporal) hunk specified by the agent control using "windows" (see boxes in this figure), pointing to different fragments of the physical world (portions of matter) with the space coordinates $x_{1}, x_{2}, x_{3}$ and the time coordinate $t$. Perception is realized in different moments (or periods) of time $t_{1}, \ldots, t_{7}$

- interpretation and judgement of importance of phenomena taking place in her/his activity environment;

- judgement of phenomena from her/his environment (in particular, causes and consequences of the phenomena) from the perspective of her/his hierarchy of needs;

- construction, initialization, realization, verification, adaptation, and termination of interaction plans;

Fig. 1 The c-granule intuition

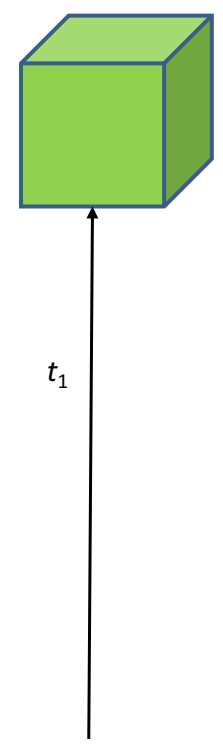

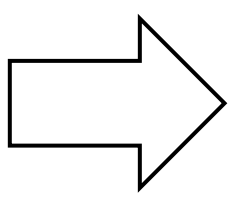

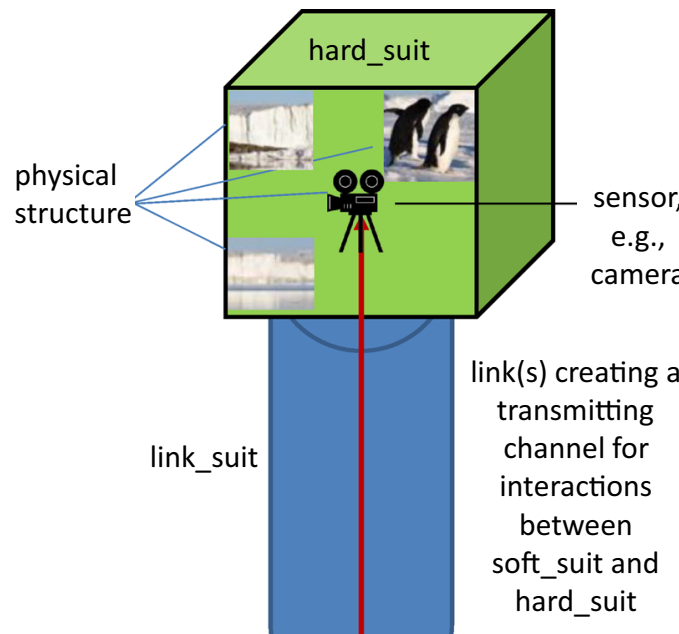

details

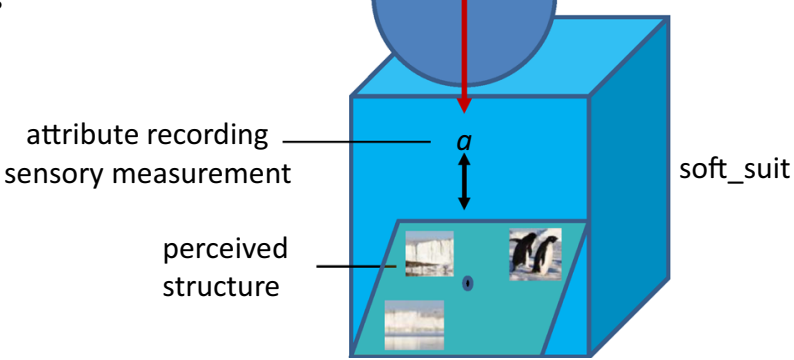


- communication, cooperation and competition of ag with other agents.

Calculi of c-granules are defined starting from some elementary c-granules, e.g., granules corresponding to reading or storing measured values, simple sensory measurements, and indiscernibility or similarity of classes. The agent's control as well as the interactions with the environment, make it possible to generate new c-granules from the already defined ones. The hard_suits, link_suits, and soft_suits of more compound c-granules are defined using the relevant networks over already defined c-granules. The networks are satisfying some constraints, which can be interpreted as definitions of types of networks. The link_suits of such more compound granules are responsible for transmission of interactions between the hard suits and soft_suits represented by the corresponding networks. The results and/or properties of transmitted interactions are recorded in the soft_suits.

We assume that for any agent there is a distinguished family of her/his c-granules creating the private language of the agent (Jankowski et al. 2014; Skowron et al. 2012). Moreover, we assume that elements of the private language can be encoded by infogranules.

We also assume that information about states of some of the physical beings from the soft_suit can be decoded using expressions from the private language of the agent. For example, these can be propositional formulas over descriptors (Pawlak 1991; Pawlak and Skowron 2007) or expressions from a (simplified) fragment of natural language (Zadeh 1979, 2001). Moreover, in the soft_suit of a c-granule the interactions, which are perceived in the hard_suit through the transmitting channels from the link_suit, are recorded. This is typical for sensory measurement. On the other hand, a c-granule may cause some interactions in its hard_suit by transmitting through its link_suit some interactions from the soft_suit. However, the c-granule may perceive the results (or properties) of such interactions in the hard_suit, only using the soft_suit. This is done on the basis of the results (or properties) of these caused interactions in the hard_suit, which are transmitted through the link_suit to the soft_suit. These results (or properties) may be different from the predicted ones, which can be stored a priori in the soft_suit. This is typical for performing actions initiated by c-granules.

One should note that the process of distinguishing (or discovering) the relevant family of c-granules creating the internal language is a very complex process. The relevant infogranules are discovered in hierarchical aggregation of infogranules considered in relevant contexts. In general, such infogranules are called semiotic c-granules. Infogranules are used for constructing the target infogranules. On the basis of satisfiability (to a degree) of such target infogranules (interpreted as approximations of complex vague concepts), relevant actions are undertaken by the agent aiming to satisfy her/his needs.

An illustrative example of c-granule is presented in Fig. 3, where (i) $h_{i}$ s are hunks corresponding to the "space windows" (i.e., windows in the spatiotemporal space which are constant over the agent's time in the example) of c-granules in the network, (ii) $s_{i}$ s denote link_suits for transmitting interactions from $h_{i}$ s in the environment $E N V$ to soft_suits of c-granules in the network, (iii) $S, S^{\prime}$ are trees representing hierarchical aggregations of c-granules starting from some input c-granules to some output c-granules grounded on hunks $h, h^{\prime}$. These two hunks are encoded, respectively, by infogranules $C, C^{\prime}$ (see Fig. 3) belonging to the agent's private language, where $C, C^{\prime}$ represent approximations of complex vague concepts used for initiation of actions $\mathrm{ac}_{i}$. The states (in the context of the given c-granule $g$ ) of hunks $h, h^{\prime}$ at a given slot (moment) $t$ of the agent's time are recorded as some properties of $h, h^{\prime}$, and they are the perceived results (at the agent's time moment $t$ ) of $h, h^{\prime}$, respectively. The states are interpreted as satisfiability degrees of $C, C^{\prime}$. In this way, the perception of the current situations in the environment $E N V$ are represented. The c-granules representing actions $\mathrm{ac}_{i}$ are initiated on the basis of the satisfiability degrees of $C, C^{\prime}$ representing the currently perceived situation in the environment $E N V$.

The process of perceiving the current situation is realized first by transmitting interactions from hunks $h_{i}$ s corresponding to the "space windows" through the links $s_{i} \mathrm{~s}$, and then through link_suits in $S, S^{\prime}$ up to hunks $h, h^{\prime}$ used to represent the perceived current situation.

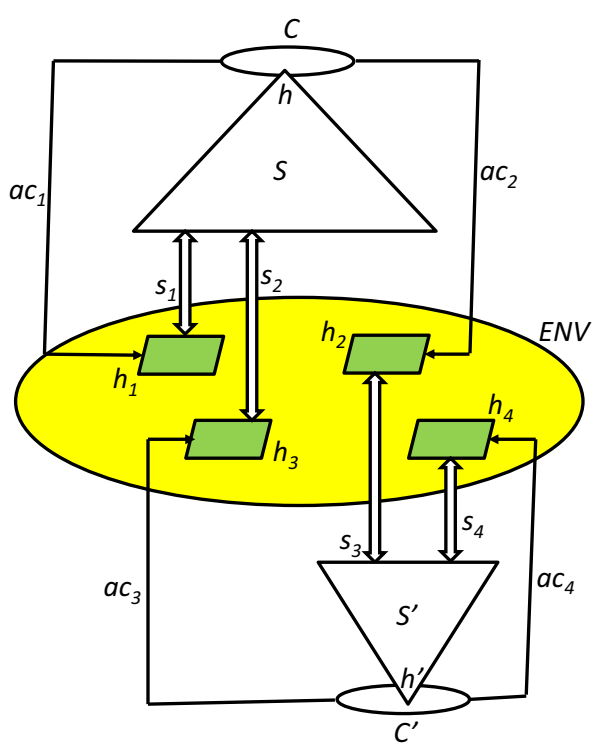

Fig. 3 An example of c-granule $g$ defined as a network (configuration) of other c-granules 
These interactions lead to changes of states of $h, h^{\prime}$. These changes are encoded by changes of degrees of satisfiability of $C, C^{\prime}$. The c-granules for actions $\mathrm{ac}_{i} \mathrm{~s}$ are responsible for initiating interactions in "space windows" $h_{i}$ s corresponding to actions $\mathrm{ac}_{i} \mathrm{~s}$. The results of the modified interactions caused by actions are transmitted through the network to $h, h^{\prime}$, which consequently leads to modification of their states.

The discussed c-granules may represent complex objects. In particular, agents and their societies can be treated as c-granules too. An example of c-granule representing a team of agents is presented in Fig. 4, where some guidelines for implementation of AI projects in the form of a cooperation scheme of different agents responsible for relevant cooperation areas are illustrated. In the figure the following members with exemplary competence areas are included:

- DATA - data acquisition, assessment, cleansing, structuring, management and governance;

- PROBLEMS-identification, classification and prioritization,

- FEATURES-feature discovery, computation and exploration up to maximally large and meaningful sets of potentially relevant features for important observed and contextual phenomena; selection of potentially high quality and small subsets of features;

- SENSORS and ACTUATORS-discovery, evolution and construction of sensors/actuators, adaptive control of sensor/actuator parameters, discovery of physical world structures and phenomena;

- DOMAIN KNOWLEDGE-acquisition, representation, management and governance of domain

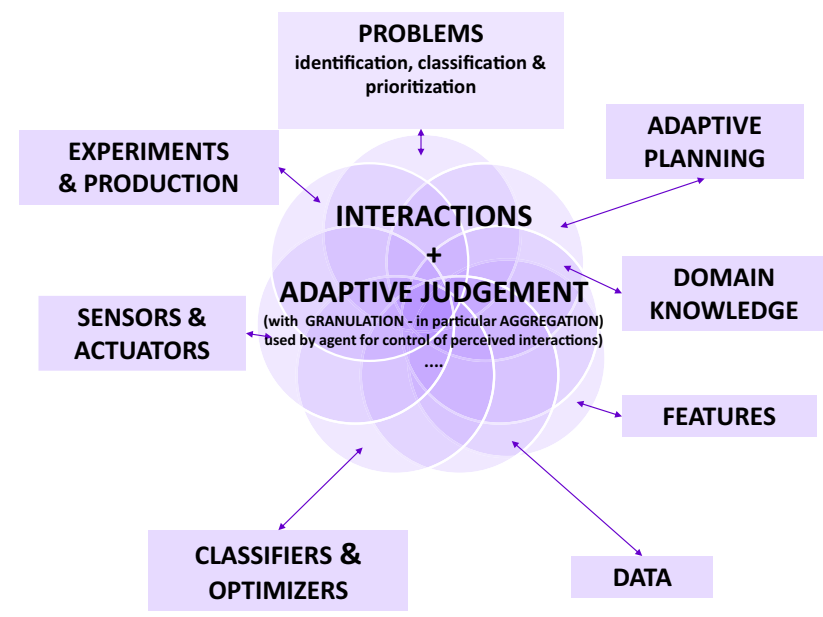

Fig. 4 Cooperation scheme of an agent team responsible for relevant competence area knowledge (e.g., ontology, rules for using language, history, applicable laws), selection of relevant knowledge for supporting solutions of prioritized tasks/ actions;

- ADAPTIVE PLANNING-problems identification and specification, tasks/ actions prioritization (based on constrains specification), planning adaptation/change control, especially change of paradigms for dealing with vague complex concepts and classifier construction;

- EXPERIMENTS-improvement of acceptance criteria computation for satisfiability degrees of vague complex concepts relevant to application requirements, scenario of testing experiments, preparation and execution experiments, results collecting and assessment;

- CLASSIFIERS and OPTIMIZERS-selection and implementation of techniques for interactive learning of vague complex concepts, including adaptation and use of inference and decision rules as arguments for or against decisions and conflict resolution-toward construction of classifier societies (and/or intelligent agents); optimizers for relevant classes of domainspecific problems (most of the classifiers, adaptation techniques and many other AI technologies depend on optimization techniques).

This cooperation scheme may be treated as a higher level c-granule. We propose to model a complex system as a society of agents. It is worthwhile mentioning that c-granules also create the basis for the construction of the agent's language of communication and the language of evolution.

An agent operates on a local world of c-granules. For achieving the target goals, the control of an agent, from the respective local world of the agent, aims at controlling computations performed on c-granules. Actions, also represented by c-granules, are used by the agent's control in exploration and/or exploitation of the environment on the way to achieve their targets. The c-granules are also used by agents for representing their perception about the interactions with the physical world. Due to the limited ability of agent's perception usually only a partial information about the interactions of the physical world may be available to the agents. Hence, in particular the results of the actions performed by agents cannot be predicted with certainty. For more details regarding IGrC based on c-granules the readers are referred to Jankowski et al. (2014) and Skowron et al. (2012).

One of the key issues of the approach related to c-granules is a kind of integration between investigations of physical and mental phenomena. This idea of integration follows from the suggestions presented by many scientists. 


\section{Interactive computations on c-granules realized by agents}

Each agent's configuration is composed out of c-granules. The agent control initializes the initial configuration. In Fig. 5 some possible components of an agent for interactions are illustrated. Among them some are:

(i) control (C),

(ii) internal memory (M),

(iii) interactions realized by the control $\mathrm{C}$ between the control granule and memory granule by means of c-granules generated by control $\mathrm{C}$ for eliciting interactions

(a) with the external environment (c-granules with parts: M, link 1-2 (1-3) and hunk H-2 (H-3)) and

(b) with internal parts of the agent other than memory $\mathrm{M}$ (c-granule with parts: $\mathrm{M}$, link $1-1$, and hunk $\mathrm{H}-1)$.

The agent's control generates new configurations of c-granules using, e.g., (i) formation of a c-granule representing the configuration, (ii) initiation of interactions in the configuration, and (iii) recording of results of interactions transmitted by links in the configuration. Note, that many activities in each of the steps may be realized concurrently (or in parallel).

It is worthwhile mentioning that contrary to the existing computation models realized by Turing machine, the results of interactions can be only predicted by the agent's control, but the results of this prediction can be in general different from the results of real interactions between agent and the environment due to uncertainty of the unpredictable environment. In particular, this may be implied by the uncertain information possessed by the agent about the

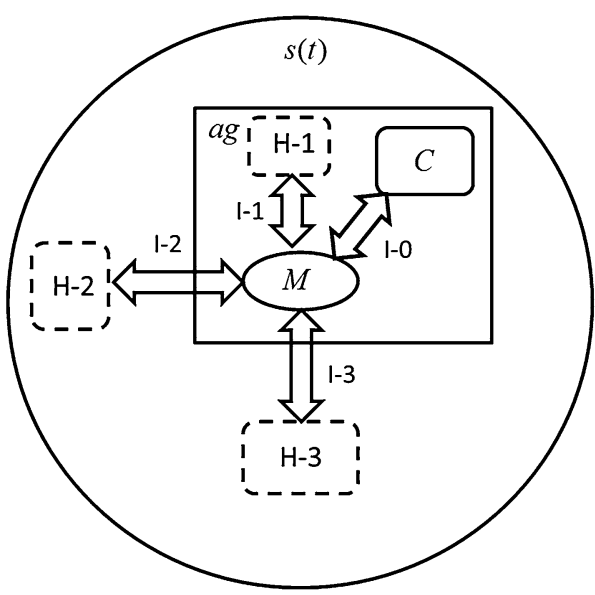

Fig. 5 Basic agent components for interactions environment, due to limitations of the available resources (e.g., sensors), which are necessary for building agent's perception strategies.

In Fig. 6, we illustrate how the abstract definition of operation from soft_suit interacts with other suits of the c-granule. It is necessary to distinguish two cases. In the case of soft_suit, the results of operation realized by the interactions of the hunks, available in the soft_suit itself, should be consistent with the specifications encoded in the link_suit. However, the result specified in the soft_suit can be treated only as an estimation of the real one which may be different due to the unpredictable interactions in the hard_suit.

Here, we would like to present a point of departure from the Stephen Kleene's proposal for (partial) recursive functions equivalent to the Turing model of computability. Kleene's definition is based on distinguishing some elementary computable functions and some distinguished operations under which the set of partial recursive functions is closed (Kleene 1936).

In our case, the selection of what is an elementary "computable" function depends on the agents ability to construct such a function in the physical world. For example, one can consider the process of computing a value of a complex function using the quantum computing paradigm presented by Andrew Chi-Chih Yao, the Turing award winner, during the panel discussion at the $2014 \mathrm{Web}$ Intelligence Congress (WIC 2014) (http://wic2014.mimuw. edu.pl/) in Warsaw. He was discussing a gedanken experiment for computing values of complex function $f(x)$ through the following steps: (i) grow a crystal $C$ tailored to $f$ and $x$, (ii) shine an optical wave on $C$, and (iii) from the diffraction pattern, figure out $f(x)$. One can take another point of view, and consider, e.g., real-valued signals or videos as elementary functions. Another example is related to the relational machine project by Ulam and Bednarek (1990), where the authors proposed to use some relations as elementary entities on which computations should be performed. They were trying to implement the idea using optical computing.

The operations of aggregation of c-granules related to such complex objects are computationally admissible, if only we can realize them in the physical world. This situation is illustrated in Fig. 6 concerning the computation of operation $\otimes$ using c-granules embedded in the physical world. In Fig. 6 there are three distinguished parts of cgranule: soft_suit, link_suit, and hard_suit. "Computability" of the value $\otimes\left(G_{1}, G_{2}\right)$ (for given $\left.G_{1}, G_{2}\right)$, firstly requires (at the proper moments of the agent's time) an access to the relevant physical configuration of objects (hard_suit) realized by interactions transmitted by links from the soft_suit to the relevant parts of the hard_suit into which representations of arguments $G_{1}, G_{2}$ are encoded. 
Fig. 6 Illustration for aggregation implementation in the physical world-

"computability" of $\otimes$ function

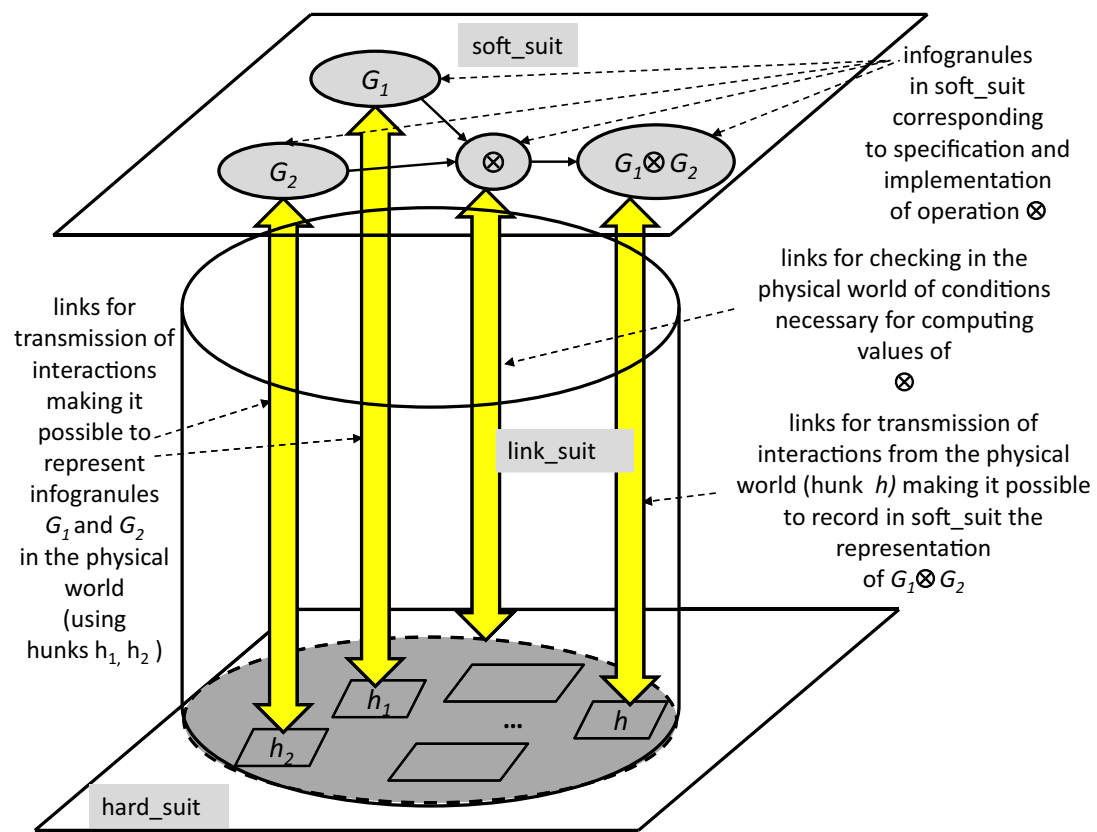

Secondly, it needs to use links to initiate relevant interactions in the hard_suit responsible for computing the value of the function $\otimes$. Finally, it needs encoding the value of $\otimes$ on arguments $G_{1}, G_{2}$ by transmitting relevant interactions (using links) from the distinguished parts of the hard_suit to the soft_suit, and encoding the results of these interactions properly in the soft_suit. Hence, the "computability" in our case depends on possibilities of perceiving and realizing in the physical world of relevant interactions.

There are some other important differences between interactive computations based on c-granules and Turing computations which will be discussed in detail elsewhere. Let us note here that the environment, over which computations on c-granules run, is unknown to the agent. The agents, through interactions with the environment, are learning how to act effectively in it. After sufficient interaction agents may gain expertise, and this is not provided a priori, but are extracted from the environment's behavior.

The reader may compare the discussed model of interactive computations with ecorithms introduced by Valiant (2013):

Unlike most algorithms, they can run in environments unknown to the designer, and they learn by interacting with the environment how to act effectively in it. After sufficient interaction they will have expertise not provided by the designer, but extracted from the environment.

The point of view, that the interactive computing on complex granules needs to be based on the process of interactions with the physical world, is important for
Natural Computing too. The agent's observation to understand such computations is dependent on the physical world (see Deutsch et al. 2000, p. 268).

The agent hypotheses about the models of computations can be verified only through interactions running in the physical world. These models should be adaptive to incorporate changes when deviations of the predicted trajectories of computations from the perceived real ones become significant.

The issues discussed in this section raise a question about the control over interactive granular computations. In the following sections, we emphasize the importance of the risk management by the agent's control.

\section{Agent control based on IGrC and related tasks}

Agents realize their goals by performing actions. Hence, it is very important to discover some measures for evaluating the correctness of a selection of a given action in a given situation. For any action $a$, one can consider a complex vague concept $Q_{a}$ representing such a measure. For a particular situation $s$, the value of $Q_{a}(s)$, is a c-granule representing the degree to which $Q_{a}(s)$ is satisfied at $s$, i.e., the correctness degree of the selection of the action $a$ at $s$. The c-granule $Q_{a}(s)$ consists of two main c-subgranules representing arguments for and against the satisfiability of $Q_{a}(s)$. These arguments are derived from the judgement based on the estimation that $a$ potentially can be initiated at the situation $s$ with respect to the efficiency management (Jankowski et al. 2014; Skowron et al. 2012). For example, in the risk assessment (ISO 31000 standard. http:// 
webstore.ansi.org/) the goal of the judgement is to identify the main risks. On the basis of the risk degrees another judgement, called the risk treatment, is performed. Some modifications of performed actions, called controls (or new controls), are considered against the existing (or possible) vulnerabilities. These new controls could suggest of avoiding the risk, reducing the risk, removing the source of the risk, modifying consequences, changing probabilities, sharing the risks with other agents, retaining the risk or even increasing the risk to pursue the opportunity (see http://www.praxiom.com/iso-31000-terms.htm).

In a relevant fragment of natural language, one should judge the degrees of satisfiability of $Q_{a}(s)$ for all relevant actions. One should also judge conflict among the degrees corresponding to different actions to select the best action(s) for execution at a given situation.

One can consider the above-mentioned tasks of approximation of action guards as the task of complex game discovery (see Fig. 7) from data and domain knowledge in cooperation with the domain experts.

The discovery process of complex games, in particular complex vague concepts which are embedded in them, often is based on hierarchical learning supported by domain knowledge (see, e.g., Bazan 2008). An agent is interacting with the environment for discovering the concepts and the cause-effect relationships relevant for the complex games. These concepts and relationships are used by the agent to judge the results of interactions for efficient initiation of relevant actions. It is also worthwhile

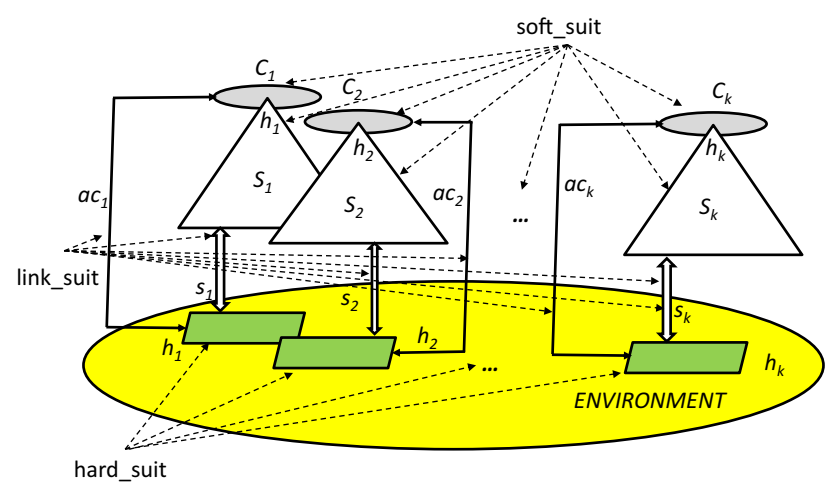

Fig. 7 Complex game represented by a c-granule. This c-granule consists of three other networks soft_suit, link_suit and hard_suit. The soft_suit consists of c-granules corresponding to hierarchical schemes $S_{1}, \ldots, S_{k}$ used for generating computational building blocks for approximation of complex vague concepts $C_{1}, \ldots, C_{k}$ representing guards for initiation of actions (or plans) $\mathrm{ac}_{1}, \ldots, \mathrm{ac}_{k}$. Judgement of satisfiability degrees of guards is used for initiation of actions. The actions as well as links $s_{1}, \ldots, s_{k}$ of hierarchical schemes to the corresponding hunks $h_{1}, \ldots, h_{k}$ create link_suit. The hard_suit consists of hunks $h_{1}, \ldots, h_{k}$. Agents are using actions to control interactions and, in consequence, the computations over granules are progressing due to interactions mentioning that these games are evolving with time (drifting with time) together with the data and knowledge about the approximated concepts as well as with the relevant strategies for adaptation of games used by agents. Hence, adaptive strategies are required for enabling agents to control their behavior to achieve the targets. It is also to be noted that these strategies should be learned from the available uncertain data and domain knowledge.

Let us summarize our considerations on the idea of discovery of games. Decision-making under uncertainty involves large number of complex vague concepts. Among them some are concepts related to identification of the current situation, discovery of the relevant context relative to which one should consider the actual situation (by considering the past, the possible future, including risks, costs or benefits), discovery of similarity measures of the current situation (or plans) with the observed ones in the past, discovery of relevant concepts for measuring the deviation degrees of the predicted situation with the real one. Moreover, for dealing with complex systems there is a need for a language in which adaptive judgement over concepts, relevant for these systems, could be performed. In particular, let us mention the need for adaptive judgement for conflict resolution among the arguments for and against concerning the satisfiability of these concepts. The reader is also referred here to the paradigm of Computing with Words (CWW) (see, e.g., Zadeh 1996, 2001, 2012; http://www.cs.berkeley.edu/ zadeh/presentations.html, and Sect. 6). The sentences made by Pearl (Pearl 2009) (see Sect. 5.6) and the paradigm of Perception-Based Computing (PBC) (e.g., Skowron and Wasilewski 2010; Zadeh 2001, 2012) are also relevant ideas to be referred to. One of the basic tasks of PBC is hierarchical learning of complex vague concepts used for comprehending the perceived situations. Let us recall some sentences from (Skowron and Wasilewski 2010) to explain that PBC is related to IGrC:

Perception is characterized by sensory measurements and ability to apply them to reason about satisfiability of complex vague concepts used, e.g., as guards for actions or invariants to be preserved by agents. Such reasoning is often referred as adaptive judgement. Vague concepts can be approximated on the basis of sensory attributes rather than defined exactly. Approximations usually need to be induced using hierarchical modeling. [...] Unfortunately, discovery of structures for hierarchical modeling is still a challenge. On the other hand, it is often possible to acquire or approximate them from domain knowledge.

For real-life projects it is hardly possible to expect that the high-quality models of the discussed complex vague 
concepts can only be induced on the basis of automatic methods without acquiring the agents' domain knowledge through cooperation with the domain experts.

One natural direction is to construct dialogue systems different from the traditional data mining systems. In the future, it will be then possible for users to formulate hypotheses, which the systems may verify interacting through a dialogue with the users. Such systems will allow us for more efficient discoveries. One can predict that such systems will be widely used in other domains too.

However, several challenges need to be resolved before such systems get used widely. In particular, they are related to the ontology of (complex vague) concepts, and relations among them on which agents can base for problem solving. Moreover, one should consider a language in which adaptive judgement about satisfiability of these concepts and relations can be performed. A challenge is to transfer the ontology and the language to the system so that the system becomes able to perform the necessary judgements with satisfactory quality.

In the study by Skowron and Jankowski (2015) and Skowron et al. (2015), a discussion illustrating how rich such ontology can be, and how complex tasks are to be solved using judgement is presented. Agents are perceiving a part of the open physical world, and they are interacting with the perceived world. Concept and relations postulated in the study by Skowron and Jankowski (2015) and Skowron et al. (2015) create the key ontological basis for WisTech (Jankowski and Skowron 2007). There are several groups of postulates. Some of them are related to the physical character of the agent, c-granules and interaction models, while the others concern the efficiency management of judgements and the realization of the prioritized needs of the agent. The postulates are specifying some basic concepts which are important for interactive computations on complex granules realized by agents for achieving their goals. It is worthwhile mentioning that in (Skowron and Jankowski 2015; Skowron et al. 2015) only a general preliminary framework for applications in reallife (intelligent) systems was formulated. There is a need for further work to make this specification more detailed and precise.

\section{IGrC and problem solving}

One of the important challenges for the IGrC development is to get scalable methods for data analytics (Jagadish et al. 2014) including (i) scalable techniques for data management, relative to different classes of problems from different domains, as well as (ii) efficient hybridization and integration of relevant techniques relative to the domain of applications (expressed, e.g., by specification of the class of problems to be solved). Let us note that scalability cannot be achieved without collective wisdom. Hence, the important area for the further development of the WisTech program arises.

In this section, a preliminary discussion on some main tasks, which should be supported by IGrC dealing with Big Data, is included. Among them, two are as follows.

1. Filtering Big Data relative to the user's view expressed by the higher level primitives.

2. Filtering Big Data relative to the user's view about specific problem (or class of problems).

The first task is related to the relevant ontology development for the considered domain, as well as to the methods of transferring it to systems for further use. Nowadays, there are many available tools for designing ontologies. Methods for approximation of ontology have been also developed (see, e.g., Bazan 2008; Nguyen et al. 2004) making it possible to transfer ontology approximation to the systems. Then, the system may use approximated concepts and relations for generating new granules (e.g., new features or patterns) relevant for the approximation of complex vague concepts. However, further work is needed for making these methods scalable for Big Data and domain knowledge.

In the following sections, we give some comments on the issues related to the second task.

\subsection{Problem specification by users}

In this section, we discuss issues related to the specification of problems, which may be faced by users for developing systems based on IGrC and Big Data.

Let us consider the following challenge mentioned in Jagadish et al. (2014):

If users are to compose and build complex analytical pipelines over Big Data, it is essential they have appropriate high-level primitives to specify their needs.

Let us observe that the above-mentioned high-level primitives are often complex vague concepts, which are semantically "far away" from the raw data. Hence, to make such concepts available by the system it is necessary to develop methods for constructing (inducing) high-quality classifiers for such concepts. The problem of specification, given by an user, is defined over such concepts.

The user's task may be to deliver the relevant granules representing complex objects, satisfying the specification to a satisfactory degree (Polkowski and Skowron 2001). Such granules are discovered and/or constructed using the hierarchical approach, where relevant strategies are to search for relevant granules through granulation and 
degranulation processes. The delivered granules may be treated as computational building blocks for approximation of complex vague concepts representing the user's specification. These approximations represent how the system is comprehending the user's specification. We have already justified that the process of inducing such approximations is challenging.

It is also worthwhile mentioning that approximations of concepts (such as concepts related to comprehending the user higher level primitives or some expressions over them describing the situation and/or user needs) related to perception are induced by the system with the help of actions.

For example, in the context of reasoning about changes of situation, one should take into account that the predicted actions or/and plans may depend not only on the changes of past situations but also on the performed actions (or plans) in the past. This is strongly related to the idea of perception pointed out in Noë (2004):

The main idea of this book is that perceiving is a way of acting. It is something we do. Think of a blind person tap-tapping his or her way around a cluttered space, perceiving that space by touch, not all at once, but through time, by skillful probing and movement.

This is or ought to be, our paradigm of what perceiving is.

On the basis of the partial understanding of the user's specification, the system may deliver some proposals for solutions. Next, the user may add some comments on them, which in turn may help in improving or reconstructing the delivered granules. The system should be able to "understand" these comments and search for the granules more relevant to the user's specification. A continuation of such a dialogue between user and the system should lead to a satisfactory solution corresponding to the user's requirements. Moreover, the whole "dialogue trajectory" should have an acceptable quality. The acceptability criterion could depend on the consumption of time in a dialogue for reaching a satisfactory solution. This means that the system should control the schemes of computation for achieving the target goal.

One can treat the above-discussed case of problems as a special case of checking satisfiability of complex vague concepts. These concepts can be interpreted as guards for initiation of actions or plans by the agent (see Sect. 4). In the discussed example related to Big Data, these actions may represent users' reactions on the solutions proposed by the system. In this more general case, the granules constructed by the system are interpreted as the degrees (representing arguments for and against) of satisfiability of complex vague concepts. Let us note that the system should be equipped with strategies for resolving conflicts between these arguments for and against.
In the next section, we present some approaches which appear to be very useful in searching for relevant calculi of granules and particular granules from the families of granules defined by these calculi.

\subsection{Construction and discovery of relevant granules}

In $\mathrm{GrC}$, we create calculi of granules by specifying elementary granules (e.g., indiscernibility or similarity classes) and some operations constructing new granules from the already defined ones (Skowron and Stepaniuk 2004). In this section, we briefly outline some of the approaches for new granule generation. For a given problem, one should discover a relevant calculi of granules, and deliver a method of searching for relevant granules (in a selected calculi) which could be used as computational building blocks for approximation of vague concepts used in the problem specification. These vague concepts may represent guards of actions or plans performed by an agent. The actions are initiated on the basis of the judgement of satisfiability degrees of these guards in a given situation.

We start from granule aggregation defined by join operations with the constraints over information systems (Skowron and Stepaniuk 2005). This approach allows us to generate new infogranules of different types. Some of these infogranules are giving rise to new information systems. These systems can be used for generation of new infogranules such as indiscernibility or similarity classes of granules of a given type, new attributes or features, classifiers, clusters, and other patterns. In the case of c-granules methods for aggregation of c-granules can be obtained in an analogous way. However, discovery of relevant aggregations becomes harder. We also explain how aggregations of granules can be used in modeling self-organization process of agents. Through self-organization, new kinds of granules are generated. Then, we discuss how discovery of a relevant hierarchy of the basic logical tools, namely satisfiability relations, can be used for new granule generation. We also discuss interaction of granules realized through dialogues of agents. Such interactions are leading towards generation of new granules relevant for agents. Important classes of granules are related to private and social languages of agents. Strategies for granule generation by self-organization and communication of agents are especially important for complex adaptive systems, where the goal is to obtain relevant emergent behavioral patterns satisfying a given specification to a satisfactory degree (Desai 2005; Liu 2001).

We also emphasize the role of risk management in controlling computations performed by agents over c-granules. Finally, we discuss a special kind of reasoning called adaptive judgement used by the agent's control for reasoning about granules and computations over them. This 
reasoning is also based on constructions over relevant granules.

\subsubsection{Context, structural objects, and self-organization}

One of the important problems in hierarchical learning of the approximations of complex vague concepts is the discovery of relevant contexts on different levels of hierarchical learning. Contexts can be modeled by aggregation of information (decision) systems based on the join operations with their respective constraints (Skowron and Stepaniuk 2005) (see Fig. 8). Cartesian product of the universes of aggregated information systems is filtered by constraints. Constraints are specifying the structure of objects on the new hierarchical level obtained by aggregation. The structure is defined by relations over the vectors of attribute values from the aggregated information systems. Constraints can also be treated as specification of types of objects in the aggregated information systems. For more details, the readers are referred to (Skowron and Stepaniuk 2005).

In the discussed case, there are two groups of (conditional) attributes in information (decision) systems. The values of attributes from the first group are fixed by the agent's control while the values of attributes from the other group are the results of a function of values of attributes from the first group and interactions with environments. As an instance, let us consider that parameters of sensors or actions, which need to be activated, belong to the first group of attributes. Then the sensory measurements, based on the values from the first group and interactions with environments, constitute the values of the attributes of the second group.

Top-down decomposition strategies of specification create such schemes with the help of which construction of relevant patterns can be discovered. Let us consider an example of decomposition of information systems with the type of objects characterized by relation $R$ over tuples of attribute-value vectors into two information systems with object types characterized by relations $R_{1}, R_{2}$. The

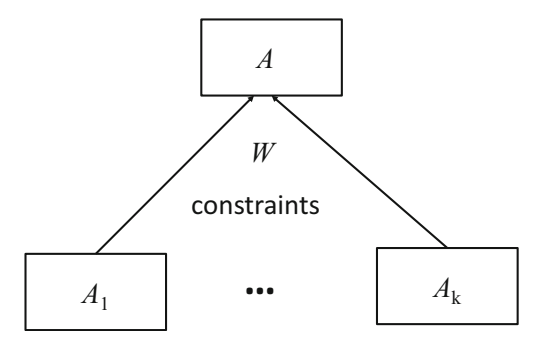

Fig. 8 Join with constraints $W$ of information systems $A_{1}, \ldots, A_{k}$ to information system $A$. The constraints are used for filtering relevant tuples of objects from the Cartesian product of the object universes of $A_{1}, \ldots, A_{k}$, e.g., those which are sufficiently close or similar corresponding join operation with constraints specifies a construction of $R$ from $R_{1}, R_{2}$. One can ask if such a construction can be modeled using simple local interactions only. For example, such local interactions may concern dependencies between values of attributes from the group of attributes defined by the control of neighboring agents, i.e., they can be fixed by neighboring agents. Here, we assume that the values of control attributes lying in the neighborhood of the first agent are perceived by the other agents. Searching for relevant contexts under simple constraints can be feasible. However, one should consider that such simplified searching not always can give the relevant aggregated constraints. One can observe here an analogy with the 13th Hilbert problem:

Can every continuous function of 3 variables be written as a composition of continuous functions of 2 variables?

and the result by Vitushkin from 1954:

There are continuously differentiable functions of 3 variables which are not the superposition of continuously differentiable functions of 2 variables.

The discussed problem is related to the self-organization process initiated from local interactions and leading to global emergent patterns.

The issues of self-organization have been intensively studied for years (e.g., Estep 2014; Pfeifer et al. 2007). Methods based on self-organization are crucial for dealing with Big Data, and further research is required in this regard.

Let us refer here once again to (Pfeifer et al. 2007) (p.1088):

\section{[...] viewing an [...] agent [...] as a complex} dynamical system enables us to employ concepts such as self-organization and emergence rather than hierarchical top-down control. [...] autonomous agents display self-organization and emergence at multiple levels: at the level of induction of sensory stimulation, movement generation, exploitation of morphological and material properties, and interaction between individual modules and entire agents.

We propose to use the top-down decompositions for generation of decomposition schemes, along which discovery of agent's self-organization may proceed (e.g., aiming at discovery of relevant contexts or object structures for the generation of relevant emergent patterns). These schemes are making the discovery process of selforganization feasible by bottom-up realization using the top-down decomposition schemes acquired from users. However, one should also note that the decomposition schemes generated in the top-down decomposition create 
only hypotheses. Hence, searching for discovery of relevant decompositions will require backtracking. Further development of methods for discovery of self-organization still requires much more work. Here, we would like to mention only the important interactions in this learning process of top-down decompositions with bottom-up selforganization.

\subsubsection{Satisfiability and new granules}

Let us observe that the satisfiability relations in the IGrC framework can be treated as tools for constructing new information granules. In fact, for a given satisfiability relation, the semantics of formulas relative to this relation is defined. In this way the candidates for new relevant information granules are obtained. We would like to emphasize on this very important feature that the relevant satisfiability relation for the considered problems, is not given but it should be induced (discovered) on the basis of a partial information encoded in the respective information (decision) systems. For real-life problems, it is often necessary to discover a hierarchy of satisfiability relations before we obtain the relevant target level. Information granules constructed at different levels of this hierarchy finally lead to relevant ones for the approximation of complex vague concepts represented by complex granules expressed in natural language (see Fig. 9).

Let us discuss some examples of c-granules constructed over a family of satisfiability relations being at the disposal of a given agent. This discussion has some roots in intuitionism (see, e.g., Martin-Löf 1984). Let us consider a remark made by Martin-Löf (1984) about judgement presented in Fig. 10.

In the approach based on c-granules, the judgement for checking values of descriptors (or more compound formulas) pointed by links from simple c-granules is based on interactions of some physical parts considered over time and/or space (called hunks) and pointed by links of c-granules. The judgement for the more compound c-granules is defined by a relevant family of procedures also realized by means of interactions with the physical parts.

Let us explain the above claims in more detail.

Let us assume that a given agent ag has at the her (his) disposal a family of satisfiability relations

$\left\{\models_{i}\right\}_{i \in I}$,

where $\models_{i} \subseteq \operatorname{Tok}(i) \times \operatorname{Type}(i), \operatorname{Tok}(i)$ is a set of tokens and Type $(i)$ is a set of types (using the terminology from Barwise and Seligman 1997). The indices of satisfiability relations are vectors of parameters related to time, space, spatiotemporal features of physical parts represented by hunks, or actions (plans) to be realized in the physical world.

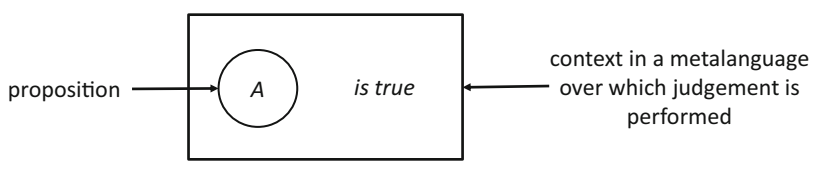

Fig. 10 Judgement of truth in a metalanguage: "When we hold a proposition to be true, then we make a judgement" (Martin-Löf 1984)
Fig. 9 Interactive hierarchical structures [gray arrows show interactions between hierarchical levels and the environment, arrows at hierarchical levels point from information (decision) systems representing partial specifications of satisfiability relations to induced from them theories consisting of rule sets]

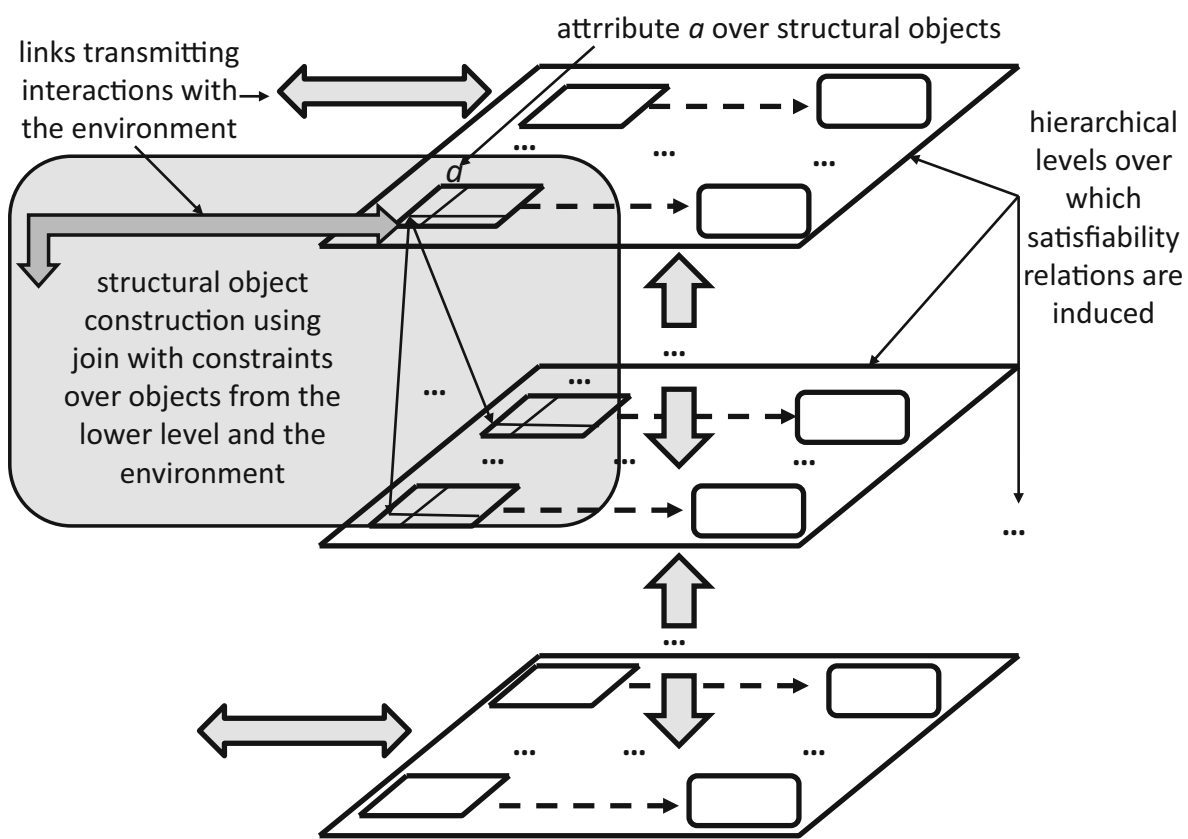


In the discussed example of elementary c-granules, $\operatorname{Tok}(i)$ is a set of hunks and, Type $(i)$ is a set of descriptors (elementary infogranules) (Pawlak and Skowron 2007), respectively, pointed by the link represented by $\models_{i}$. The procedure for computing the value of $h \models_{i} \alpha$, where $h$ is a hunk and $\alpha$ is an infogranule (e.g., descriptor or formula constructed over descriptors), is based on the interaction of $\alpha$ with the physical world represented by the hunk $h$.

The agent's control can aggregate some simple c-granules into more compound c-granules. For example, by selecting some constraints on subsets of $I$ it is possible to select relevant sets of simple c-granules generated by $\models_{i}$ over these subsets, and consider them as new, more compound c-granules. For example, constraints over values of descriptors pointed by links of elementary c-granules can be taken into account, and sets of descriptors selected by such constraints can be used to create more compound c-granules. Values of new descriptors (pointed by links of these more compound granules) are computed by new procedures. The computation process again is realized through interaction with the physical parts represented by hunks, which are pointed by links of c-granules, included in the compound c-granule. Moreover, in a procedure for computing the values of more compound descriptors the values of the descriptors included in the elementary c-granules (of the considered more compound c-granule) are used. It is to be noted that this procedure is also realized in the physical world with the help of the relevant interactions.

In hierarchical modeling aiming at inducing relevant c-granules (e.g., for approximation of complex vague concepts), one can consider so far constructed c-granules as tokens. For example, they can be used to define structured objects representing corresponding hunks, and using new satisfiability relations (from a given family) they can be linked to the relevant higher order descriptors together with the appropriate procedures (realized by interactions of hunks) for computing values of these descriptors. This approach generalizes hierarchical modeling developed for infogranules (see, e.g., Bazan 2008; Nguyen et al. 2004) in the context of hierarchical modeling of c-granules, which is important for many real-life projects.

We have assumed before that the agent ag is equipped with a family of satisfiability relations. In the framework of $\mathrm{GrC}$, based on c-granules, satisfiability relations are tools for constructing new c-granules. In fact, for a given satisfiability relation, the semantics of descriptors (and more compound formulas) relative to this relation can be defined.

However, in real-life cases the situation is more complicated. The agent ag should have strategies for discovering new relevant satisfiability relations on the way of searching for target goals (solutions of problems). For example, the approximations of concepts and relations from the ontology discovered on lower levels of the hierarchy of satisfiability relations should make it possible to generate the relevant computational building blocks for inducing on the next hierarchy levels, e.g., the model of similarity relation between the treatment plans (delivered by medical expert, and predicted by the decision support system) (Bazan 2008). Searching for relevant satisfiability relations is related to adaptive judgement.

\subsubsection{Comments on dialogues of agents in IGrC}

In this section, we present some preliminary comments on dialogues among agents. Dialogues of agents from a given team can lead to a common understanding of the problems of concern and help to get a cooperative problem solving strategy. The issues related to reasoning based on dialogues are not trivial, especially when one would like to propose a treatment incorporating the possibility of combining different dominating paradigms of reasoning in logic. This point of view was well expressed by Johan van Benthem in Rahwan and Simari (2009) (see Foreword, p.viii):

I see two main paradigms from Antiquity that come together in the modern study of argumentation: Platos Dialogues as the paradigm of intelligent interaction, and Euclids Elements as the model of rigor. Of course, some people also think that formal mathematical proof is itself the ultimate ideal of reasoning - but you may want to change your mind about reasonings peak experiences' when you see top mathematicians argue interactively ats a seminar.

Dialogues enable the agents to (efficiently) search for solutions. Very often a query, formulated in IGrC by an agent, involves vague concepts from natural language; e.g., one can consider queries given by an user to a dialoguebased search engine. Agents are expecting to receive c-granules satisfying their specifications to some satisfactory degrees. The meaning of satisfiability to a degree should be learned on the basis of dialogues among agents embedded in the systems based on IGrC. Satisfiability to a degree gives some flexibility in searching for solutions. The solutions do not need to be exact. This may make the process of searching for constructions of such c-granules feasible. It is worthwhile mentioning that such constructions should be robust relative to the deviations of components.

Using dialogues, agents may try to recognize the meaning of c-granules received from the other agents. They can do this by learning approximations of received c-granules in their own languages. A given agent may acquire the description of ontology of the concepts used by another agent. However, usually a given agent can only acquire an approximation of concept-ontology possessed 
by another agent. This idea of shared knowledge among agents may be very useful in solving problems by any individual agent (see e.g., Bazan 2008; Nguyen et al. 2004). Let us note that the ontology approximation may also be used in efficient searching for relevant contexts of queries received by agents from other agents.

One of the challenges for adaptive judgement, performed by a given agent ag, is the task of learning the approximation of derivations performed by another agent $\mathrm{ag}^{\prime}$, assuming that an approximated concept-ontology of $\mathrm{ag}^{\prime}$ is already available to ag. The agent ag may approximate, to a satisfactory degree, the derivations performed by $\mathrm{ag}^{\prime}$ with the help of the constructions of solutions delivered by $\mathrm{ag}^{\prime}$.

\subsection{Risk management by agents in IGrC}

In this section, we add some comments on risk management. Let us note that practical judgement is involved in efficient management (Jankowski et al. 2014; Skowron et al. 2012).

Risk may be understood as interaction (of agents) with uncertainty (of environment). Perception of risk is a subjective judgement, which people make about the severity and/or probability of a risk. This may vary from one person to another. Any human endeavor carries some risk, but some are much riskier than the others (The Stanford Encyclopedia of Philosophy: http://plato.stanford.edu/ archives/spr2014/entries/risk/).

Since the very beginning, all human activities were done at risk of failure. We have seen the low quality of risk management in areas such as finance, economics, and many others in the recent years. In this regard, improvement in the risk management is of a particular importance for the further development of complex systems. The importance of risk management illustrates the following example from the financial sector. Many of financial risk management experts consider Basel II rules (see http://en. wikipedia.org/wiki/Basel_Committee_on_Banking_Super vision) as a causal factor in the credit bubble prior to the 2007-2008 collapse. Namely, in Basel II one of the principal factors of financial risk management was

outsourced to companies that were not subject to supervision, credit rating agencies.

Of course, now we do have a new "improved" version of Basel II, called Basel III. However, according to an OECD (see http://en.wikipedia.org/wiki/Basel_III) the medium-term impact of Basel III implementation on GDP growth is negative and estimated in the range of $-0.05 \%$ to $-0.15 \%$ per year (see also Slovik 2011).

On the basis of experience from many areas, we have now many valuable studies on different approaches to risk management. Currently, the dominant terminology is determined by the standards of ISO $31 \mathrm{~K}$ (ISO 31000 standard. http://webstore.ansi.org/). However, the logic of inferences in risk management is dominated by the statistical paradigms, especially by Bayesian data analysis initiated about 300 years ago by Bayes, and regression data analysis initiated about 200 years ago by Legendre and Gauss. They initiated many detailed methodologies specific for different fields. A classic example is the risk management methodology in the banking sector, based on the recommendations of Basel II standards for mathematical models of risk management. The current dominant statistical approach is not satisfactory because it does not give effective tools for inferences about the vague concepts and relations between them.

A particularly important example of a vague relation in risk management is the relation of a cause-effect dependencies between various events. It should be noted that the concept of risk in ISO $31 \mathrm{~K}$ is defined as the effect of uncertainty on objectives. Thus, by definition, the vagueness is also an essential part of the risk concept.

A slightly modified version of sentences of Pearl (2009) for risk management can be presented as follows: traditional statistical approach to risk management inference is strong in devising ways of describing data and inferring distributional parameters from sample. However, in practice risk management inference requires two additional ingredients:

- a science-friendly language for articulating risk management knowledge, and

- a mathematical machinery for processing that knowledge, combining it with data and drawing new risk management conclusions about a phenomenon.

As very accurately specified by the Turing test, adding both the above-mentioned components is an extremely difficult task, and relates to the core of AI research. In the context of our applications, the idea of Turing test boils down to the fact that on the basis of a "conversation" with a hidden risk management expert and a hidden machine one will not be able to distinguish who is the man and who is the machine.

We propose to extend the statistical paradigm by adding the two above-discussed components for designing the high-quality risk management systems based on IGrC.

For the risk management based on IGrC one of the most important task is to develop strategies for inducing approximations of the vague complex concepts involved in the domain of concern of the risk management. Let us note that the approximations are providing methods for checking their satisfiability (to a degree). A typical example of such vague concept is the statement of the form: "now we do have a very risky situation". Among such concepts, the complex vague concepts representing the role of guards, on 
which the activation of actions performed by agents are based, are of special importance.

These vague complex concepts are represented by the agent's hierarchy of needs. In the risk management, one should consider a variety of complex vague concepts and relations between them, as well as the reasoning schemes related to the bow tie diagram (Skowron et al. 2012).

\section{Adaptive judgement}

The reasoning which is used to support problem solving, makes it possible to derive relevant c-granules, and control interactive computations over c-granules for achieving the target goals, is called an adaptive judgement.

Adaptive judgement over interactive computations is a mixture of reasoning based on deduction, abduction, and induction. In particular, case-based reasoning, analogybased reasoning, reasoning from experience, reasoning based on observed changes in the environment, or reasoning with application of meta-heuristics from natural computing are used. One should note that judgment figures in the explanation of behavior, in inference, and in experience. Hence, the theory of judgment has roots in psychology, in logic, and in phenomenology (Martin 2006). In particular, the meaning of practical judgement goes beyond typical tools for reasoning based on deduction or induction (Thiele 2010):

Practical judgement is not algebraic calculation. Prior to any deductive or inductive reckoning, the judge is involved in selecting objects and relationships for attention and assessing their interactions. Identifying things of importance from a potentially endless pool of candidates, assessing their relative significance, and evaluating their relationships is well beyond the jurisdiction of reason.

For example, a particular question for the agent's control concerns discovering strategies for models of dynamic changes of the agent's attention. This may be related to discovery of changes in a relevant context necessary for the judgement.

Let us note that the intuitive judgement and the rational judgement are distinguished as different kinds of judgement.

Among the tasks for adaptive judgement there are the following ones supporting reasoning towards,

- inducing relevant classifiers (e.g., searching for relevant approximation spaces, discovery of new features, selection of relevant features (attributes), rule induction, discovery of inclusion measures, strategies for conflict resolution, adaptation of measures based on the minimum description length principle),

- prediction of changes,

- initiation of relevant actions or plans,

- discovery of relevant contexts,

- adaptation of different sorts of strategies (e.g., for existing data models, quality measures over computations realized by agents, objects structures, knowledge representation and interaction with knowledge bases, ontology acquisition and approximation, hierarchy of needs, or for identifying problems to be solved according to priority of needs),

- learning the measures of inclusion between granules from sources using different languages (e.g., the formal language of the system and the user natural language) through dialogue,

- strategies for development and evolution of communication language among agents in distributed environments, and

- strategies for efficiency management in distributed computational systems.

Below we discuss some issues related to adaptive judgement in IGrC.

In some cases judgement methods may be based on formal languages, i.e., the expressions from such languages are used as labels (syntax) of granules. However, there are also paradigms such as Computing With Words (CWW), due to Professor Lotfi Zadeh (Zadeh 1996, 2001, 2012), where labels of granules are words (i.e., words or expressions from a relevant fragment of natural language), and computations are performed on words (http://www.cs.ber keley.edu/ zadeh/presentations.html). In IGrC it is necessary to develop new methods extending the approaches for approximating vague concepts expressed in natural language, and for approximating reasoning on such concepts. It is also important to note that information granulation plays a key role in implementation of the strategy of divide-and-conquer in human problem solving (Zadeh 1979, 2001). Hence, it is important to develop methods which could perform approximate reasoning along such decomposition schemes, delivered by the strategy of divide-and-conquer in human problem solving, and induce the relevant granules as computational building blocks for constructing the solutions for the considered problems.

In case of systems, based on IGrC, the users are often specifying problems in fragments of a natural language with the constraint, that their solutions will satisfy specifications to some satisfactory degrees. Hence, methods for approximation of domain ontology (i.e., ontology on which a fragment is based) as well as approximations of constructions representing solutions based on concepts from 
the domain ontology should be developed. The rough set approach in combination with other soft computing approaches is used for approximation of the vague concepts (Bazan 2008). These approximations may help the system to follow, in an approximate sense, the judgement schemes expressed in the relevant (for the considered problems) fragment of a natural language. It is worthwhile to emphasize here the importance of dialogues between users and system in the process of obtaining the relevant approximations.

Very often the problems related to systems based on IGrC concern control tasks. Examples of control tasks may be found in different areas, such as the medical therapy support, management of large software projects, algorithmic trading or control on unmanned vehicles, to name a few. Such projects are typical for ULS. Any of such exemplary projects is supported by (large) data and domain knowledge distributed over computer networks and/or Internet. Moreover, interactions of agents with the physical world, which are often unpredictable, are unavoidable. Computations performed by agents are aiming at constructing, learning, or discovering granules, which in turn makes it possible to understand the concerned situation (state) to a satisfactory degree. The relevant control of computations based on understanding of the situation is realized using approximations of complex vague concepts playing the role of guards, responsible for initiation of actions (or plans) by agents. In particular, different kinds of granules, discovered from data, are used for constructing these approximations. The main processes, namely granulation and degranulation, characterize, respectively, the synthesis and decomposition of granules in the process of constructing relevant resultant granules.

The efficiency management in controlling the computations in IGrC are of great importance for the successful behavior of individuals, groups or societies of agents. In particular, such efficiency management is important for constructing systems based on large data for supporting users in problem solving. The efficiency management covers risk assessment, risk treatment, and cost/beneft analysis. The tasks related to this management are related to control tasks aiming at achieving the high-quality performance of (societies of) agents. One of the challenges in efficiency management is to develop methods and strategies for adaptive judgement related to adaptive control of computations. The efficiency management in decision systems requires tools to discover, represent, and access approximate reasoning schemes (ARSs) (over domain ontologies) representing the judgement schemes (Bazan 2008; Skowron and Stepaniuk 2004). ARSs are approximating, in a sense, judgements expressed in relevant fragments of simplified natural language. Methods for inducing of ARSs are still under development. The systems for problem solving are enriched not only by approximations of concepts and relations from ontologies but also by ARSs.

We would like to stress that still much work should be done to develop approximate reasoning methods about complex vague concepts for the progress of the development of IGrC, in particular for the efficiency management in systems based on IGrC. This idea was very well expressed by Leslie Valiant (see, e.g., http://en.wikipedia. org/wiki/Vagueness, http://people.seas.harvard.edu/ vali ant/researchinterests.htm; Valiant 2013):

\section{A fundamental question for artificial intelligence is to characterize the computational building blocks that are necessary for cognition. A specific challenge is to build on the success of machine learning so as to cover broader issues in intelligence. [...] This requires, in particular a reconciliation between two contradictory characteristics - the apparent logical nature of reasoning and the statistical nature of learning.}

It should be noted that the approach based on IGrC is well suited for implementation. This was proved, e.g., in inducing c-granules [such as clusters, time windows or their sequences, similarity neighborhoods, processes, classifiers (Skowron and Stepaniuk 2005), see also Sect. 5.4] in hierarchical learning (see, e.g., Bazan 2008; http://www. mimuw.edu.pl/ bazan/roughice/?sLang=en). The induced c-granules are used as computational building blocks for approximation of high-level specifications (often expressed in natural language) or complex games (see Sect. 4). On different levels of hierarchy, c-granules are used to represent structural objects and also as features (attributes) over such objects (see Sect. 5.4). Also methods for discovery of c-granules in the form of approximate reasoning schemes were developed (see, e.g., Skowron and Stepaniuk 2004 and Sect. 6). It is worthwhile mentioning that there is a need for developing methods for inducing complex networks of c-granules relevant for more complex tasks. For example, actions or plans should be embedded into hierarchies of c-granules what leads to feedback loops in the networks. Such networks of c-granules (which are also c-granules!) aim at preserving some invariants (over time) of complex systems, e.g., representing the requirements for high security of the system. Further development of IGrC foundations will bring methods for inducing c-granules being complex networks in which also physical objects (such as robots or different units) and humans exist. This is very important for development of ULS systems, in particular for CPSs and W2T. This will help to deal with challenges for such systems (Lamnabhi-Lagarrigue et al. 2014). 


\section{Conclusions}

The approach for modeling interactive computations based on c-granules is presented, and its importance for the efficiency management of controlling interactive computations over c-granules is outlined. It is worthwhile mentioning that in modeling and/or discovering granules, tools from different areas are used. Among these areas some are machine learning, data mining, multi-agent systems, complex adaptive systems, logic, cognitive science, neuroscience, and soft computing. IGrC is aiming at developing a unified methodology for modeling and controlling computations over complex objects, called c-granules, as well as for reasoning about such objects and computations over them. In particular, such a methodology is of great importance for ULS.

The discussed concepts such as interactive computation and adaptive judgement are among the basic ingredients in the field of WisTech. Let us mention here the WisTech meta-equation:

\section{WISDOM $=$ INTERACTIONS + ADAPTIVE JUDGEMENT + KNOWLEDGE.}

The presented approach has a potential for being used for developing computing models in different areas, such as natural computing (e.g., computing models for metaheuristics or computing models for complex processes in molecular biology), computing in distributed environments under uncertainty realized by multi-agent systems (e.g., in social computing), modeling of computations for feature extraction (constructive induction) used for approximation of complex vague concepts, hierarchical learning, discovery of planning strategies or strategies for coalition formation by agents as well as for approximate reasoning about interactive computations based on such computing models. In our research, we plan to further develop the foundations of interactive computations based on c-granules. The approach will be used for the development of models of computations and their analysis in Natural Computing (Rozenberg et al. 2012), W2Ts (Zhong et al. 2013), CPSs (Lamnabhi-Lagarrigue et al. 2014), and ULS (Cyber-physical and ultra-large-scale systems 2013).

Acknowledgments This work by Andrzej Skowron and Andrzej Jankowski was partially supported by the Polish National Science Centre (NCN) Grants DEC-2011/01/D/ST6/06981, DEC-2012/05/B/ ST6/03215, DEC-2013/09/B/ST6/01568 as well as by the Polish National Centre for Research and Development (NCBiR) under the Grant O ROB/0010/03/001. Soma Dutta was supported by the ERCIM postdoc fellowship.

Open Access This article is distributed under the terms of the Creative Commons Attribution 4.0 International License (http:// creativecommons.org/licenses/by/4.0/), which permits unrestricted use, distribution, and reproduction in any medium, provided you give appropriate credit to the original author(s) and the source, provide a link to the Creative Commons license, and indicate if changes were made.

\section{References}

Bargiela A, Pedrycz W (eds) (2003) Granular Computing: An Introduction. Kluwer Academic Publishers, Dordrecht

Barwise J, Seligman J (1997) Information Flow: The Logic of Distributed Systems. Cambridge University Press, Cambridge

Bazan J (2008) Hierarchical classifiers for complex spatio-temporal concepts. In: Transactions on Rough Sets IX: Journal Subline. LNCS, vol 5390, pp 474-750

Cyber-physical and ultra-large scale systems (2013). http://resources. sei.cmu.edu/library/asset-view.cfm?assetid $=85282$

Desai A (2005) Adaptive complex enterprises. Commun ACM 45:32-35

Deutsch D, Ekert A, Lupacchini R (2000) Machines, logic and quantum physics. Bull Symb Log 6:265-283

Estep M (2014) Self-organizing natural intelligence: Issues of knowing, meaning, and complexity. Springer, Heidelberg

Goldin D, Smolka S, Wegner P (eds) (2006) Interactive Computation: the New Paradigm. Springer, Berlin

Heller M (1990) The Ontology of Physical Objects. Four Dimensional Hunks of Matter. Cambridge Studies in Philosophy. Cambridge University Press, Cambridge,

Jagadish H, Gehrke J, Labrinidis A, Papakonstantinou Y, Patel JM, Ramakrishnan R, Shahabi C (2014) Big data and its technical challenges. Commun ACM 57:86-94

Jankowski A, Skowron A (2007) A WisTech paradigm for intelligent systems. In: Transactions on Rough Sets VI. LNCS, vol 4374. Springer, Heidelberg, pp 94-132

Jankowski A, Skowron A, Swiniarski RW (2014) Interactive complex granules. Fundamenta Informaticae 133:181-196

Kleene SC (1936) General recursive functions of natural numbers. Mathematische Annalen 112:727-742

Lamnabhi-Lagarrigue F, Di Benedetto MD, Schoitsch E (2014) Introduction to the special theme cyber-physical systems. Ercim News 94:6-7

Liu J (2001) Autonomous Agents and Multi-agent Systems: Explorations in Learning, Self-organization and Adaptive Computation. World Scientific Publishing, Singapore

Martin WM (ed) Theories of Judgment. Psychology, Logic, Phenomenology. Cambridge University Press, New York (2006)

Martin-Löf P (1984) Intuitionistic Type Theory (Notes by Giovanni Sambin of a series of lectures given in Padua, June 1980). Bibliopolis, Napoli

Nguyen SH, Bazan J, Skowron A, Nguyen HS (2004) Layered learning for concept synthesis. In: Transactions on Rough Sets I: Journal Subline. LNCS, vol 3100, pp 187-208

Noë A (2004) Action in Perception. MIT Press, Cambridge

Pawlak Z (1991) Rough Sets: Theoretical Aspects of Reasoning about Data. System Theory, Knowledge Engineering and Problem Solving, vol 9. Kluwer Academic Publishers, Dordrecht

Pawlak Z, Skowron A (2007) Rudiments of rough sets. Inf Sci 177(1):3-27

Pearl J (2009) Causal inference in statistics: An overview. Stat Surv 3:96-146

Pedrycz W (2013) Granular Computing Analysis and Design of Intelligent Systems. CRC Press/Taylor \& Francis, Boca Raton

Pedrycz W, Skowron A, Kreinovich V (eds) (2008) Handbook of Granular Computing. Wiley, Hoboken 
Pfeifer R, Lungarella M, Iida F (2007) Self-organization, embodiment, and biologically inspired robotic. Science 318:1088-1093

Polkowski L, Skowron A (2001) Rough mereological calculi of granules: A rough set approach to computation. Comput Intell Int J 17(3):472-492

Rahwan I, Simari GR (2009) Argumentation in Artificial Intelligence. Springer, Berlin

Rozenberg G, Bäck T, Kok J (eds) (2012) Handbook of Natural Computing. Springer, Berlin

Skowron A, Jankowski A (2015) Interactive granular computing. In: Ciucci D, Wang G, Mitra S, Wu W (eds) International Joint Conference on Rough Sets, Tianjin, China, Korea, November 20-23, 2015, Proceedings of the RSKT 2015. Lecture Notes in Artificial Intelligence, vol 9436. Springer, Heidelberg, pp 50-61

Skowron A, Stepaniuk J (2004) Information granules and roughneural computing. In: Pal SK, Polkowski L, Skowron A (eds) Rough-Neural Computing: Techniques for Computing with Words. Cognitive Technologies, Springer, Heidelberg, pp 43-84

Skowron A, Stepaniuk J (2005) Hierarchical modelling in searching for complex patterns: Constrained sums of information systems. J Exp Theor Artif Intell 17:83-102

Skowron A, Wasilewski P (2010) An introduction to perception based computing. In: Kim TH, Lee YH, Kang BH, Ślęzak D (eds) Proceedings of FGIT 2010. Lectures Notes in Computer Science, vol 6485. Springer, Heidelberg, pp 12-25

Skowron A, Wasilewski P (2011) Information systems in modeling interactive computations on granules. Theor Comput Sci 412(42):5939-5959

Skowron A, Pal SK, Nguyen HS (eds) (2011) Special issue on rough sets and fuzzy sets in natural computing. Theor Comput Sci 412(42):5815-5973

Skowron A, Jankowski A, Wasilewski P (2012) Risk management and interactive computational systems. J Adv Math Appl $1: 61-73$

Skowron A, Jankowski A, Dutta S (2015) Toward problem solving support based on big data and domain knowledge: Interactive granular computing and adaptive judgement. In: Japkowicz N,
Stefanowski J (eds) Big Data Analysis. Studies in Big Data. Springer, Heidelberg (in print)

Slovik P, Cournède (2011) Macroeconomic impact of Basel III. Working Papers, vol 844. OECD Economics Publishing, OECD Economics Department. http://www.oecd.org/eco/Workingpapers

Thiele LP (2010) The Heart of Judgment: Practical Wisdom, Neuroscience, and Narrative. Cambridge University Press, Cambridge

Ulam S, Bednarek A (1990) On the theory of relational structures and schemata for parallel computation. In: Analogies between Analogies: the Mathematical Reports of S.M. Ulam and his Los Alamos Collaborators, pp 477-508

Valiant L (2013) Probably Approximately Correct. Nature's Algorithms for Learning and Prospering in a Complex World. Basic Books. A Member of the Perseus Books Group, New York

Yao YY (2008) A unified framework of granular computing. In: Pedrycz W, Skowron A, Kreinovich V (eds) Handbook of granular computing. Wiley, Hoboken, pp 401-410

Zadeh LA (1979) Fuzzy sets and information granularity. In: Advances in Fuzzy Set Theory and Applications. North-Holland, Amsterdam, pp 3-18

Zadeh LA (1996) Fuzzy logic = computing with words. IEEE Trans Fuzzy Syst 4:103-111

Zadeh LA (1997) Toward a theory of fuzzy information granulation and its centrality in human reasoning and fuzzy logic. Fuzzy Sets Syst 90:111-127

Zadeh LA (2001) A new direction in AI: Toward a computational theory of perceptions. AI Mag 22(1):73-84

Zadeh A (2012) Computing with Words: Principal Concepts and Ideas. In: Studies in Fuzziness and Soft Computing, vol 277. Springer, Heidelberg

Zhong N, Ma JH, Huang R, Liu J, Yao Y, Zhang YX, Chen J (2013) Research challenges and perspectives on Wisdom Web of Things (W2T). J Supercomput 64:862-882 